\title{
Familial versus Sporadic Breast Cancer: Different Treatments for Similar Tumors?
}

\author{
Ellen G. Engelhardt'1, Mieke Kriege'2, Maartje J. Hooning2', Caroline Seynaeve², \\ Rob A. E. M. Tollenaar ${ }^{3}$, Christina J. van Asperen ${ }^{4}$, Margreet G. E. M. Ausems ${ }^{5}$, \\ Lonneke V. van de Poll-Franse ${ }^{6}$, Stella Mook ${ }^{7}$, Senno Verhoef ${ }^{8}$, Matti A. Rookus ${ }^{1}$, \\ HEBON Collaborators ${ }^{9}$, Marjanka K. Schmidt1,10 \\ ${ }^{1}$ Division of Psychosocial Research and Epidemiology, Netherlands Cancer Institute, Amsterdam, \\ The Netherlands \\ ${ }^{2}$ Family Cancer Clinic, Department of Medical Oncology, Erasmus MC-Daniel den Hoed Cancer Centre, \\ Rotterdam, The Netherlands \\ ${ }^{3}$ Department of Surgery, Leiden University Medical Centre, Leiden, The Netherlands \\ ${ }^{4}$ Department of Clinical Genetics, Leiden University Medical Centre, Leiden, The Netherlands \\ ${ }^{5}$ Department of Medical Genetics, University Medical Centre Utrecht, Utrecht, The Netherlands \\ ${ }^{6}$ Eindhoven Cancer Registry, Comprehensive Cancer Centre South, Eindhoven, The Netherlands \\ ${ }^{7}$ Department of Radiation Oncology, Netherlands Cancer Institute, Amsterdam, The Netherlands \\ ${ }^{8}$ Family Cancer Clinic, Netherlands Cancer Institute, Amsterdam, The Netherlands \\ ${ }^{9}$ Hereditary Breast and Ovarian Cancer Netherlands (HEBON) Collaborators (see end of article for full list of \\ HEBON collaborators and affiliations) \\ ${ }^{10}$ Division of Molecular Pathology, Netherlands Cancer Institute, Amsterdam, The Netherlands \\ Email: mk.schmidt@nki.nl
}

Received 14 July 2015; accepted 27 September 2015; published 30 September 2015

Copyright (C) 2015 by authors and Scientific Research Publishing Inc.

This work is licensed under the Creative Commons Attribution International License (CC BY).

http://creativecommons.org/licenses/by/4.0/

(c) (i) Open Access

\section{Abstract}

Objective: It is unclear if and to what extent family history of breast/ovarian cancer or BRCA1/2mutation carriership influences breast cancer treatment strategy. We investigated whether treatment differed between patients from $B R C A 1 / 2$ families and those unselected for family history. Methods: We included $478 B R C A 1 / 2$-related patients referred for genetic testing before or after diagnosis. Two references were used: 13,498 population-based and 6896 hospital-based patients. Surgical treatment and adjuvant chemotherapy use was analyzed using logistic regression models, stratified by tumor size, nodal status, age at and period of diagnosis, and estrogen receptor status (ER). Results: $B R C A 1 / 2$ cases aged $35-52$ years at diagnosis and/or with tumors $<2$ cm were more likely to have undergone a modified radical mastectomy (Odd Ratios (OR) ranging from 2.8 to 5.1) compared to the references. This effect was most pronounced in patients treated after 1995 (OR 5.7 to 10.3). Compared to the reference groups, chemotherapy was more often administered to BRCA1 and ER-negative BRCA1/2-cases irrespective of age and nodal status (OR 1.9 
to 24.3). Conclusion: After 1995 treatment of BRCA1/2-associated patients consisted notably of more mastectomies and adjuvant chemotherapy than their population-based counterparts with the same tumor characteristics. There is a need to be aware of such differences in daily practice and interpretation of survival studies on $B R C A 1 / 2$ mutation carriers.

\author{
Keywords \\ BRCA1/2, Familial, Breast Cancer, Treatment, Adjuvant Chemotherapy, Mastectomy, Breast \\ Conserving Therapy
}

\title{
1. Introduction
}

$B R C A 1$ and BRCA2 mutation carriers have an increased risk of developing breast cancer at a young age. These mutations confer a lifetime-risk of $50 \%-80 \%$ whereas the breast cancer risk of the general Dutch population is $13 \%$ [1] [2]. Approximately $2 \%$ - 3\% of all breast cancer cases can be attributed to a mutation in the BRCA1 or $B R C A 2$ gene [3].

Nowadays the most important prognosticators of long-term (i.e. $\geq 10$-year) survival in sporadic breast cancer patients are still the traditional factors such as tumor size, nodal status, tumor grade, age at diagnosis and ER status [4]. Current evidence suggests that these prognostic factors are also relevant for survival of BRCA2-related breast cancer patients, while they are possibly less strong prognosticators for BRCA1-related breast cancer [5]-[7]. It is still under debate whether survival in BRCA1 carriers is different from that of non-carriers. One out of two reviews based on limited evidence concluded that BRCA1 carriers have a worse overall and progression-free survival time compared to non-carriers [8] [9]. Current guidelines on breast cancer treatment do not take genetic status into account [10] [11].

In the Netherlands, breast cancer treatment guidelines for local therapy (surgery/radiotherapy) existed on a regional level as far back as the early 1970's. Breast conserving surgery was introduced in the mid-eighties following the publication of the European Organization for Research and Treatment of Cancer (EORTC) 10801 trial [12], while the first national Dutch guideline regarding adjuvant systemic therapy was implemented by the Dutch Institute for Healthcare Improvement [13] in 1998 following the presentation of the meta-analysis by the Early Breast Cancer Trialists' Collaborative Group (EBCTCG) [14], initiating a wider use of adjuvant systemic therapy most notably for node negative breast cancer [14] [15]. Continuous improvements in breast cancer treatment over the past decades, including local and systemic adjuvant therapy and the introduction of breast cancer screening, have played an important role in improving long-term breast cancer survival [16] [17].

In the Netherlands, Family Cancer Clinics were established in the early 1990's, and after the identification of the breast cancer susceptibility genes BRCA1 and BRCA2 in 1994 and 1995, respectively, referral for genetic testing and counselling became more frequent. Nowadays, breast cancer genetic counselling has become common practice in the Netherlands. Especially female breast cancer patients diagnosed at a young age ( $<35$ years) or those with a (extensive) family history of breast and/or ovarian cancer are referred for genetic testing [18]. The uptake of preventive surgery by women with a BRCA1/2 mutation in the Netherlands-with $35 \%$ opting for prophylactic mastectomy and $49 \%$ for salpingo-oophorectomy - is relatively high compared to other western countries [19]-[21]. Several studies have shown that preventive salpingo-oophorectomy not only reduces the risk of developing ovarian/fallopian tube cancer, but also reduces the breast cancer risk in BRCA1/2-mutation carriers by $50 \%-72 \%$; whereas bilateral prophylactic mastectomy reduces the risk of breast cancer by approximately 90\% - 95\% [21]-[24]. Furthermore, prophylactic salpingo-oophorectomy has been shown to improve the overall and cancer-specific survival, including breast cancer-specific survival, in BRCA1/2-mutation carriers [21] [23] [25], while data on a beneficial effect of preventive mastectomy on survival are not yet available.

While the influence of genetic status or family history of breast/ovarian cancer on the decision making regarding prophylactic surgery (i.e. prophylactic mastectomy or salpingo-oophorectomy) has been the subject of several studies, it has never been explored whether a family history of breast/ovarian cancer and/or knowledge of BRCA1/2-carriership affect choices for breast cancer treatment [26]. Therefore the aim of the current study was to determine whether patients from BRCA1 and BRCA2 families received more extensive breast cancer treatment compared to sporadic breast cancer cases. 


\section{Methods}

\subsection{Patient Selection and Data Collection}

For the current case-case study, which was conducted as part of the Hereditary Breast and Ovarian cancer Netherlands (HEBON) Resource study, females in BRCA1/2 families diagnosed with breast cancer between 1980 and 2007 were identified through the Gene-Environment Research in Hereditary Breast and Ovarian cancer Netherlands (GEO-HEBON) database [27]. All breast cancer patients who underwent genetic counselling and were (partly) treated at the Antoni van Leeuwenhoek hospital, Amsterdam, Erasmus MC-Daniel den Hoed cancer clinic, Rotterdam, Leiden University Medical Centre or University Medical Centre Utrecht were selected from the GEO-HEBON database $(n=590)$. Cases with distant metastases at diagnosis or only a ductal carcinoma in situ were excluded $(n=112)$, leaving 366 BRCA1 and 112 BRCA2 breast cancer cases for analyses. These 478 breast cancer patients consisted of women who were genotyped as BRCA1/2 mutation carrier before breast cancer diagnosis $(\mathrm{n}=36)$, women who were genotyped as BRCA1/2 mutation carrier after breast cancer diagnosis $(\mathrm{n}=383$ ), and women who had not (yet) undergone genetic testing, but belonged to proven BRCA1/2 families and were a first degree family member of a proven mutation carrier $(=$ obligate carrier $)(\mathrm{n}=59)$. These $B R C A 1 / 2$-familial cases are all referred to as BRCA1/2 cases in this paper, keeping in mind that the majority of these patients were treated for breast cancer not yet knowing that they were a BRCA mutation carrier, as BRCAtesting started in 1995 approximately. From July 2009 until January 2010 for all eligible patients detailed information on tumor characteristics, surgical and systemic treatment for the primary breast cancer, and follow-up data regarding local recurrence, distant metastases, other primary tumors and death was extracted from medical records, the GEO-HEBON and other existing (clinical) oncology databases.

We used two reference populations, a general population-based sample of breast cancer cases from the Comprehensive Cancer Centre South database $(n=13,498)$ [28] and a hospital-based case series from the Antoni van Leeuwenhoek cancer-specialized hospital (NKI-AVL), Amsterdam ( $\mathrm{n}$ 6896), applying the same exclusion criteria as for the BRCA1/2 cases. The patients from the hospital-based series were diagnosed with breast cancer between 1987-2000 [29]. The cancer hospital-based reference group was included in addition to the population-based reference group (mainly treated in general hospitals), to account for possible differences in treatment strategy between cancer-specialized and general hospitals.

Primary breast cancer treatment included local surgery, consisting of either a modified radical mastectomy or breast conserving therapy aiming at radical excision, followed by adjuvant radiotherapy (on indication after modified radical mastectomy, and always after breast conserving therapy). Also, adequate treatment of the axillary lymph nodes was performed, consisting of either systematic axillary dissection or sentinel lymphadenectomy (SN-procedure, as of the year 2000 approximately). The clinical decision to opt for a modified radical mastectomy or breast conserving surgery was largely based on tumor size (in combination with tumor location and breast volume). Adjuvant systemic therapy (i.e. chemotherapy and/or hormonal therapy) was administered after local therapy depending on the patient's age/menopausal status ( $\leq 52$ years premenopausal, $>52$ years postmenopausal), tumor stage (tumor size, and nodal status), and later also depending on tumor characteristics (e.g. differentiation grade and hormone receptor status). Since the latter part of the 1990s hormone receptor status, mainly ER status, became an important discriminating factor regarding the type of systemic treatment; a positive ER status was recognized as a predictive factor for efficacy of endocrine therapy, while in case of negative ER status only chemotherapy was considered [13] [30].

\subsection{Ethical Standards}

The HEBON Resource study was approved by the Review Board of the NKI-AVL, and according to Dutch law, no further institutional Review Board approval was needed. The use of all data in this manuscript, including that of the two control cohorts, complies with Dutch laws and follows the Scientific Codes published by the Dutch federation of Biomedical Scientific Societies [31].

\subsection{Statistical Analysis}

In order to quantitatively assess breast cancer treatment, we divided treatment into two dichotomous variables, namely 1) type of surgical treatment (modified radical mastectomy versus breast conserving therapy) and 2) use of adjuvant chemotherapy (yes versus no). Only $10 \%$ of BRCA1/2 cases had received both chemotherapy and 
hormonal therapy.

Odds ratio's for undergoing a modified radical mastectomy (versus breast conserving therapy) and for receiving chemotherapy were calculated using multivariate logistic regression analyses for BRCA1 and BRCA2 cases compared to population- and cancer hospital-based reference groups. A priori we expected the BRCA1/2 cases to be younger and to have different tumor characteristics. Based on these differences, but also based on covariates, which might influence breast cancer treatment decisions, we decided for which characteristics we would stratify and/or adjust our analyses. Logistic regression models for surgery were stratified for tumor size $(\leq 2 \mathrm{~cm}$ and $>2$ $\mathrm{cm})$ age at breast cancer diagnosis $(<35,35-52,>52)$ and period of breast cancer diagnosis $(\leq 1995,>1995$ (introduction of BRCA1/2 mutation testing in the Netherlands) and $\leq 1998,>1998$ (paradigm shift in adjuvant treatment). Analyses for chemotherapy were stratified for nodal status (negative, positive), age at breast cancer diagnosis $(<35,35-52,>52)$ and period of breast cancer diagnosis $(\leq 1995,>1995$ and $\leq 1998,>1998)$. Additionally the logistic models for chemotherapy were also run for ER negative cases only (with BRCA1 and BRCA2 patients combined due to small numbers). In the logistic regression models for surgery and for chemotherapy, covariates included were differentiation grade (1, 2, 3, and missing), nodal status (node negative, node positive, and missing), tumor size ( $\leq 2 \mathrm{~cm},>2 \mathrm{~cm}$, and missing), estrogen receptor status (positive, negative, and missing), age at breast cancer diagnosis (<35 years, $35-52$, and $>52$ ), unless the model was stratified for that factor. In the analyses for BRCA1 cases versus the reference groups, stratified for multiple factors, only cases with grade 3 tumors were included since grade 1 and grade 2 tumors are rare in BRCA1 cases. All statistical analyses were performed using SPSS Inc.18. A two-sided p of $<0.05$ was considered significant.

\section{Results}

Of the $478 B R C A 1 / 2$ breast cancer patients included, 419 were identified as BRCA1 $(\mathrm{N}=323)$ or $B R C A 2(\mathrm{~N}=$ 96) mutation carriers themselves, and 59 obligate mutation carrier. Breast cancer was diagnosed before 1995 in 256 (54\%) of the BRCA1/2 cases and after 1995 in 222 (46\%). Of the 222 BRCA1/2 cases diagnosed after 1995, $36(16 \%)$ were aware of their own carriership status and 16 (7\%) were aware of a BRCA1 or BRCA2 mutation in their family at breast cancer diagnosis and treatment.

Characteristics of BRCA1/2 cases and of the two reference groups are shown in Table 1. Mean age at breast cancer diagnosis was 41 and 44 years for BRCA1 and BRCA2 patients, respectively, compared with 57 years and 55 years for the population-based and hospital-based reference groups, respectively. The majority of the BRCA1 and BRCA2 patients had grade 3 tumors (88\% and 69\%, respectively), while this was only 32\% and 31\% for the population-based and hospital-based reference groups, respectively. The majority of the BRCA1 tumors were ER negative (77\%) compared with 25\% of the BRCA2 tumors and 23\% of the tumors in both reference groups.

We had data on prophylactic surgery for 408 of the 478 BRCA1/2 cases; 24 of them underwent a prophylactic salpingo-oophorectomy and 56 a mastectomy within 1 year of the diagnosis of the primary tumor (no data available for reference groups).

\subsection{Type of Surgery}

Figure 1 shows unadjusted trends in chemotherapy and surgical treatment per year. Type of surgical treatment by tumor size and nodal status for the two reference groups is shown in Table 2(a) and for the BRCA1/2 cases in Table 2(b). Within the BRCA1/2 cases having a tumor $\leq 2 \mathrm{~cm}, 64 \%$ of those identified as mutation carrier prior to their breast cancer diagnosis underwent a modified radical mastectomy compared to 35\% of BRCA1/2 cases who had not yet been referred for genetic testing. For the population- and hospital-based reference groups these percentages were $27 \%$ and $20 \%$, respectively. BRCA1/2 cases with a tumor $\leq 2 \mathrm{~cm}$ more often had a mastectomy and received chemotherapy (53\%) than the general population- and hospital-based reference groups (<30\%) (Table 2(c)). So, BRCA1/2 cases with tumors $\leq 2 \mathrm{~cm}$ underwent a modified radical mastectomy 1.6 to 3.1 times more often than the population- and hospital-based cases, respectively (Table 3), while there were no significant differences in type of surgery between BRCA1/2 cases and the reference patients with tumors $>2 \mathrm{~cm}$. After stratification for age at diagnosis, BRCA1/2 cases aged 35 - 52 years were significantly more likely to undergo a modified radical mastectomy compared to the reference population cases. If we stratified for tumor size and age at diagnosis (only enough cases for the BRCA1 subgroup), the higher percentage of modified radical mastectomy in BRCA1 cases only remained significant in the subgroup of patients diagnosed between 35 - 52 years and having a tumor $\leq 2 \mathrm{~cm}$. In patients treated before 1995, we did not observe a higher rate of modified radical 
Table 1. Population characteristics.

\begin{tabular}{|c|c|c|c|c|}
\hline & \multicolumn{2}{|c|}{ Clinical genetic centers } & \multicolumn{2}{|c|}{ Reference groups } \\
\hline & BRCA1 & $B R C A 2$ & Population based & Hospital based \\
\hline \multicolumn{5}{|l|}{ Medical centers, patients (N) } \\
\hline Antoni van Leeuwenhoek hospital & 79 & 30 & NA & 6896 \\
\hline Erasmus MC- Daniel den Hoed Cancer Center & 212 & 44 & NA & NA \\
\hline Leiden University Medical Center & 51 & 36 & NA & NA \\
\hline University Medical Center Utrecht & 24 & 2 & NA & NA \\
\hline Comprehensive Cancer Center South & NA & NA & 13498 & NA \\
\hline Total (N) & 366 & 112 & 13498 & 6896 \\
\hline \multicolumn{5}{|l|}{ Diagnosis year (N (\%)) } \\
\hline Range & 1980-2006 & 1981-2006 & $1980-2007$ & $1987-2000$ \\
\hline $1980-1990$ & $110(30)$ & $35(31)$ & $3860(28)$ & $1541(22)$ \\
\hline $1991-2000$ & $202(55)$ & $49(44)$ & $5112(38)$ & 5355 (78) \\
\hline 2001-2010 & $54(15)$ & $28(25)$ & $4526(34)$ & 0 \\
\hline \multicolumn{5}{|l|}{ Age at diagnosis (N (\%)) } \\
\hline Mean age (range) & $41(19-72)$ & $44(31-81)$ & $57(20-81)$ & $55(23-81)$ \\
\hline$<35$ years & $108(30)$ & $16(14)$ & $359(3)$ & $266(4)$ \\
\hline $35-52$ years & $211(58)$ & $76(68)$ & $4782(35)$ & $2998(43)$ \\
\hline$>52$ years & $47(12)$ & $20(18)$ & $8357(62)$ & $3632(53)$ \\
\hline \multicolumn{5}{|l|}{ Differentiation grade (N (\%)) } \\
\hline Grade 1 & $1(<1)$ & $4(6)$ & $1523(22)$ & 1053 (19) \\
\hline Grade 2 & $29(12)$ & $17(25)$ & $3091(46)$ & $2724(50)$ \\
\hline Grade 3 & $210(88)$ & $46(69)$ & $2168(32)$ & $1727(31)$ \\
\hline Missing & 126 & 45 & 6716 & 1392 \\
\hline \multicolumn{5}{|l|}{ Tumor diameter (N (\%)) } \\
\hline$\leq 2 \mathrm{~cm}$ & $172(52)$ & $51(52)$ & $7064(54)$ & $3529(52)$ \\
\hline$>2 \mathrm{~cm}$ & $157(48)$ & $47(48)$ & $6087(46)$ & 3320 (48) \\
\hline Missing & 37 & 14 & 347 & 47 \\
\hline \multicolumn{5}{|l|}{ Estrogen receptor (N (\%)) } \\
\hline Negative & $189(77)$ & $19(25)$ & 2196 (23) & 1050 (23) \\
\hline Positive & $56(23)$ & $57(75)$ & 7443 (77) & 3536 (77) \\
\hline Missing & 121 & 36 & 3859 & 2310 \\
\hline \multicolumn{5}{|l|}{ Nodal status (N (\%)) } \\
\hline Negative & $225(66)$ & $49(47)$ & $6624(55)$ & 3143 (48) \\
\hline Positive & 115 (34) & $56(53)$ & $5348(45)$ & 3435 (52) \\
\hline Missing & 26 & 7 & 1526 & 318 \\
\hline
\end{tabular}

NA = not applicable.

mastectomy in BRCA1/2 cases with a tumor $\leq 2 \mathrm{~cm}$ compared to the reference groups, while BRCA1 cases with tumors $\leq 2 \mathrm{~cm}$ diagnosed after 1995 had higher odds of undergoing a modified radical mastectomy compared to the population-based (OR = 5.8, 95\% CI 3 - 11.2) and the hospital-based cohort (OR = 10.3, 95\% CI 4.8 - 22.3) (Table 3). The analyses stratified for prior or post 1998 showed similarly increased odds for undergoing modified radical mastectomy in the BRCA1/2 cases diagnosed after 1998 (data not shown).

\subsection{Adjuvant Chemotherapy}

Administration of chemotherapy in relation to negative or positive nodal status is shown in Table 2(a) and Table 2(b) for the reference and BRCA1/2 groups, respectively. Chemotherapy was more often administered in 
Trends in surgery for $B R C A 1 / 2$ cases
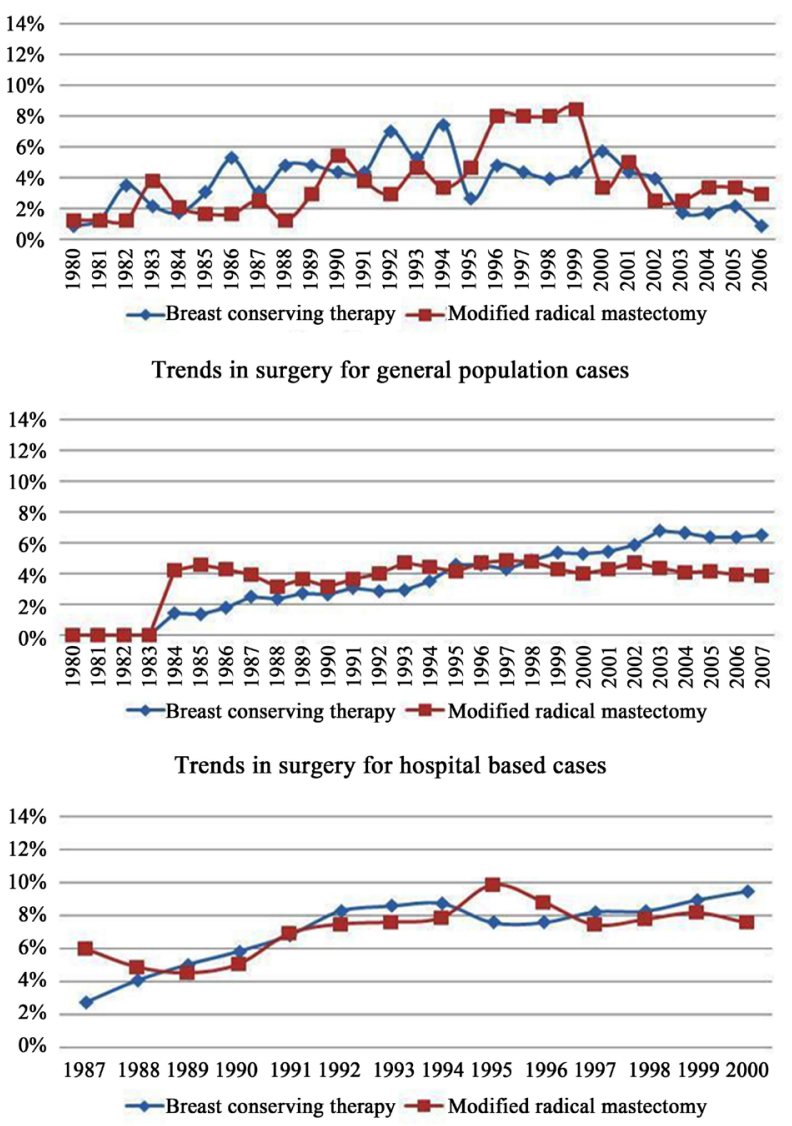

Trends in adjuvant chemotherapy for BRCA $1 / 2$ cases

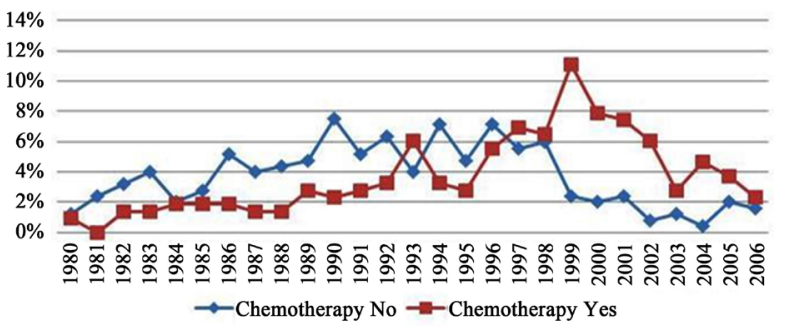

Trends in adjuvant chemotherapy for general population cases

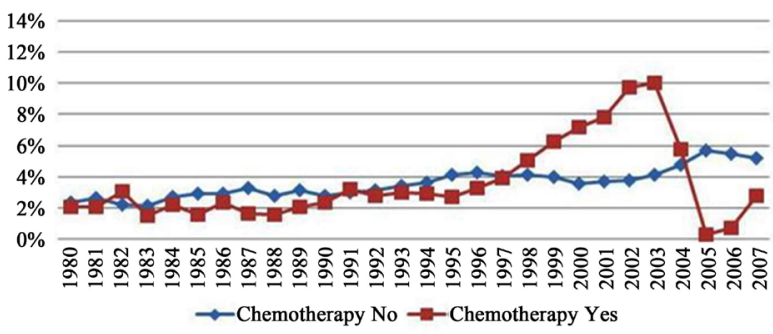

Trends in adjuvant chemotherapy for hospital based cases

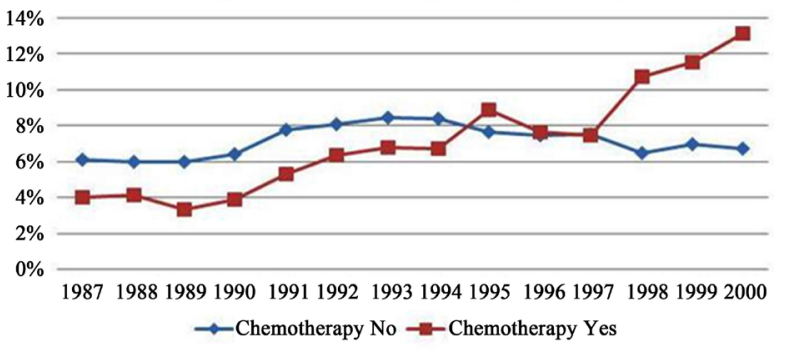

Figure 1. Trends in surgical and adjuvant chemotherapy for population subgroups.

node-negative BRCA1/2 patients genotyped prior to their breast cancer diagnosis (73\%) compared to BRCA1/2 patients who had not yet been tested (17\%; Table 2(b)); at least partly related to the fact that more chemotherapy was given in later years of diagnosis. BRCA1 and ER-negative (BRCA1and BRCA2 combined) cases across all ages and irrespective of lymph node involvement had higher odds of receiving chemotherapy compared to both reference groups while a higher odds for of receiving chemotherapy in BRCA2 cases was especially observed in the node positive subgroup and in the subgroups of 35 - 52 years and $>52$ years at diagnosis (Table 4). The higher odds of receiving chemotherapy in BRCA1/2 cases compared with the reference groups was observed in patients treated before as well as after 1995, but numbers were too small for adjusted analyses (Table 4). Node positive BRCA1 cases treated both prior to and after 1998 had increased odds of receiving chemotherapy compared to the population-based cases (OR $=5,95 \%$ CI 2.4 - 10.2; OR = 4.1, 95\% CI 1.2 - 14.2). Moreover, node negative BRCA1 cases treated after 1998 also showed significantly increased odds of receiving chemotherapy compared to the population reference group $(\mathrm{OR}=13.1,95 \% \mathrm{CI} 5.8$ - 29.4) (other data for this comparison not shown). In the current study data regarding hormonal therapy was also collected, however, the numbers of BRCA1/2 patients receiving adjuvant hormonal therapy were too small (57 cases) to perform separate analyses.

\section{Discussion}

Overall we found that BRCA1/2 cases more often underwent a modified radical mastectomy, especially BRCA1 and BRCA2 cases diagnosed after 1995 with tumours $\leq 2 \mathrm{~cm}$, and more often received adjuvant chemotherapy, especially in BRCA1 cases and/or ER-negative tumors, compared to unselected breast cancer cases with similar tumor characteristics from hospital- and population-based reference groups. These findings highlight the importance of taking type of treatment into account when comparing survival between mutation carriers and sporadic cases, something which is rarely done by studies published so far, as demonstrated by a recent systematic review 
Table 2. (a)Type of surgery and administration of chemotherapy for the reference groups; (b) Type of surgery and administration of chemotherapy by tumor subgroup in relation to timing of DNA testing; (c) Type of surgery and administration of chemotherapy by nodal status for each study population.

(a)

\begin{tabular}{|c|c|c|c|c|}
\hline & & Population-based $^{1}$ & Hospital-based $^{1}$ & $\mathrm{P}^{2}$ \\
\hline \multicolumn{5}{|c|}{ Type of surgery (N (\%)) } \\
\hline \multirow{2}{*}{$\leq 2 \mathrm{~cm}$} & Breast conserving therapy & $4758(73)$ & $2649(80)$ & \multirow{2}{*}{$<0.001$} \\
\hline & Modified radical mastectomy & $1836(27)$ & $643(20)$ & \\
\hline \multirow{2}{*}{$>2 \mathrm{~cm}$} & Breast conserving therapy & $1803(35)$ & $1240(41)$ & \multirow{2}{*}{$<0.001$} \\
\hline & Modified radical mastectomy & $3324(65)$ & 1793 (59) & \\
\hline \multicolumn{5}{|c|}{ Adjuvant chemotherapy $(N(\%))$} \\
\hline \multirow{2}{*}{ Node negative } & Yes & $277(4)$ & $132(4)$ & \multirow{2}{*}{0.967} \\
\hline & No & $6347(96)$ & $3011(96)$ & \\
\hline \multirow{2}{*}{ Node positive } & Yes & $1773(33)$ & 1477 (43) & \multirow{2}{*}{$<0.001$} \\
\hline & No & $3575(67)$ & $1958(57)$ & \\
\hline
\end{tabular}

${ }^{1}$ Numbers of patients varies due to missing values.

${ }^{2} \mathrm{P}$ values from Chi-square tests; because these are unadjusted for patient and tumor differences between the references populations, these should not be interpreted as differences in treatment practices between these populations.

(b)

\begin{tabular}{|c|c|c|c|c|}
\hline & & \multicolumn{2}{|c|}{ Clinical Genetic Centers } & \multirow{2}{*}{$\mathrm{P}^{3}$} \\
\hline & & $\begin{array}{l}\text { DNA test pre breast } \\
\text { cancer diagnosis }\end{array}$ & $\begin{array}{l}\text { DNA test post breast } \\
\text { cancer diagnosis }^{2}\end{array}$ & \\
\hline \multicolumn{5}{|c|}{ Type of surgery $(N(\%))$} \\
\hline \multirow{2}{*}{$\leq 2 \mathrm{~cm}$} & Breast conserving therapy & $10(36)$ & $106(65)$ & \multirow{2}{*}{0.011} \\
\hline & Modified radical mastectomy & $18(64)$ & $57(35)$ & \\
\hline \multirow{2}{*}{$>2 \mathrm{~cm}$} & Breast conserving therapy & $10(43)$ & $58(39)$ & \multirow{2}{*}{0.441} \\
\hline & Modified radical mastectomy & $13(57)$ & $89(61)$ & \\
\hline \multicolumn{5}{|c|}{ Adjuvant chemotherapy $(N(\%))$} \\
\hline \multirow{2}{*}{ Node negative } & Yes & $24(73)$ & $34(17)$ & \multirow{2}{*}{$<0.001$} \\
\hline & No & $9(27)$ & $169(83)$ & \\
\hline \multirow{2}{*}{ Node positive } & Yes & $14(88)$ & $107(81)$ & \multirow{2}{*}{0.677} \\
\hline & No & $2(12)$ & $25(19)$ & \\
\hline
\end{tabular}

${ }^{\mathbf{1}}$ DNA test pre breast cancer diagnosis means that either the case herself or a family member had undergone BRCA genetic testing and received the results of the test prior to the woman included being diagnosed with breast cancer.

${ }^{2}$ Numbers of patients varies due to missing values

${ }^{3} \mathrm{P}$ values from Chi-square tests; because these are unadjusted for patient and tumor differences between the pre-and post-breast cancer diagnoses tested carriers, these should not be interpreted as differences in treatment practices between those groups.

(c)

\begin{tabular}{|c|c|c|c|c|c|c|}
\hline & \multicolumn{2}{|c|}{ Treatment } & \multicolumn{3}{|c|}{ Population } & \multirow[b]{2}{*}{$\mathrm{P}^{1}$} \\
\hline & Adjuvant chemotherapy & Type of surgery (N (\%)) & BRCA $1 / 2$ cases & $\begin{array}{c}\text { General } \\
\text { population-based }\end{array}$ & $\begin{array}{c}\text { Cancer } \\
\text { hospital-based }\end{array}$ & \\
\hline \multirow{4}{*}{ Node negative } & \multirow{2}{*}{ No } & Breast conserving therapy & $130(63)$ & $3488(62)$ & $2223(81)$ & \multirow{2}{*}{$<0.001$} \\
\hline & & Modified radical mastectomy & $75(37)$ & $2114(38)$ & $531(19)$ & \\
\hline & \multirow{2}{*}{ Yes } & Breast conserving therapy & $30(45)$ & $144(55)$ & $90(71)$ & \multirow{2}{*}{0.001} \\
\hline & & Modified radical mastectomy & $36(55)$ & $118(45)$ & $36(29)$ & \\
\hline \multirow{4}{*}{ Node positive } & \multirow{2}{*}{ No } & Breast conserving therapy & $6(21)$ & $1330(43)$ & 702 (39) & \multirow{2}{*}{0.008} \\
\hline & & Modified radical mastectomy & 22 (79) & $1799(57)$ & $1087(61)$ & \\
\hline & \multirow{2}{*}{ Yes } & Breast conserving therapy & $50(36)$ & $697(44)$ & $609(44)$ & \multirow{2}{*}{0.155} \\
\hline & & Modified radical mastectomy & $90(64)$ & $884(56)$ & $780(56)$ & \\
\hline
\end{tabular}

${ }^{1} \mathrm{P}$ values from Chi-square test; because these are unadjusted for patient and tumour differences, these should not be interpreted as differences in treatment practices between groups. 
Table 3. Odds of undergoing a modified radical mastectomy versus breast conserving therapy for BRCA1/2 cases compared to population- and cancer hospital-based cases.

\begin{tabular}{|c|c|c|c|c|c|c|c|}
\hline \multirow{3}{*}{ Subgroup } & & & \multirow{3}{*}{$\mathrm{N}$} & \multicolumn{2}{|c|}{$\begin{array}{l}B R C A 1 / 2 \text { cases versus } \\
\text { population-based cases }\end{array}$} & \multicolumn{2}{|c|}{$\begin{array}{l}\text { BRCA1/2 cases versus } \\
\text { hospital-based cases }\end{array}$} \\
\hline & & & & Unadjusted & Adjusted $^{1}$ & Unadjusted & Adjusted $^{1}$ \\
\hline & & & & OR $(95 \%-C I)$ & OR (95\%-CI) & OR (95\%-CI) & OR $(95 \%-C I)$ \\
\hline \multirow{3}{*}{ BRCA 1} & \multicolumn{7}{|c|}{ Cases stratified by tumour diameter } \\
\hline & \multicolumn{2}{|c|}{$\leq 2 \mathrm{~cm}$} & 171 & $1.7(1.2-2.3)$ & $1.6(1.2-2.3)^{*}$ & $2.7(1.9-3.7)$ & $2.9(2.0-4.2)^{*}$ \\
\hline & \multicolumn{2}{|c|}{$>2 \mathrm{~cm}$} & 156 & $0.7(0.5-0.9)$ & $0.8(0.6-1.1)$ & $0.9(0.6-1.2)$ & $1.5(1.0-2.2)^{*}$ \\
\hline \multirow{2}{*}{$B R C A 2$} & \multicolumn{2}{|c|}{$\leq 2 \mathrm{~cm}$} & 51 & $2.0(1.1-3.4)$ & $1.9(1.1-3.3)^{*}$ & $3.1(1.8-5.5)$ & $3.1(1.7-5.6)^{*}$ \\
\hline & \multicolumn{2}{|c|}{$>2 \mathrm{~cm}$} & 47 & $1.2(0.6-2.1)$ & $1.3(0.7-2.4)$ & $1.5(0.8-2.7)$ & $1.9(1.0-3.7)$ \\
\hline \multirow{5}{*}{ BRCA 1} & \multicolumn{7}{|c|}{ Cases stratified by age at breast cancer diagnosis } \\
\hline & \multicolumn{2}{|c|}{$<35$} & 107 & $0.8(0.5-1.3)$ & $0.8(0.5-1.4)$ & $1.0(0.7-1.6)$ & $1.4(0.8-2.4)$ \\
\hline & \multicolumn{2}{|c|}{$35-52$} & 205 & $1.4(1.1-1.9)$ & $1.5(1.1-2.0)^{*}$ & $1.8(1.4-2.4)$ & $2.5(1.8-3.5)^{*}$ \\
\hline & \multicolumn{2}{|c|}{$>52$} & 42 & $1.3(0.7-2.4)$ & $1.2(0.6-2.3)$ & $1.6(0.9-2.9)$ & $1.8(0.9-3.7)$ \\
\hline & \multicolumn{2}{|c|}{$<35$} & 16 & $1.0(0.4-2.8)$ & $1.0(0.3-3.0)$ & $1.3(0.5-3.7)$ & $1.3(0.4-4.1)$ \\
\hline \multirow[t]{5}{*}{ BRCA 2} & \multicolumn{2}{|c|}{$35-52$} & 76 & $2.0(1.3-3.2)$ & $1.9(1.1-3.1)^{*}$ & $2.6(1.6-4.1)$ & $2.6(1.5-4.4)^{*}$ \\
\hline & \multicolumn{2}{|c|}{$>52$} & 20 & $2.3(0.9-5.6)$ & $2.4(0.9-6.8)$ & $2.7(1.1-6.7)$ & $4.4(1.5-12.9)^{*}$ \\
\hline & \multicolumn{7}{|c|}{ Cases stratified by age at breast cancer diagnosis and tumour diameter } \\
\hline & \multirow{2}{*}{$<35$} & $\leq 2 \mathrm{~cm}$ & 27 & $2.1(0.8-5.9)$ & $x^{2}$ & $1.8(0.7-4.9)$ & $x^{2}$ \\
\hline & & $>2 \mathrm{~cm}$ & 34 & $0.4(0.2-0.9)$ & $0.5(0.2-1.4)$ & $0.7(0.3-1.7)$ & $1.4(0.5-4.0)$ \\
\hline \multirow{4}{*}{$\begin{array}{l}\text { BRCA 1(only grade } \\
3 \text { tumours included }\end{array}$} & \multirow{2}{*}{$35-52$} & $\leq 2 \mathrm{~cm}$ & 63 & $2.5(1.5-4.4)$ & $2.8(1.6-4.8)^{*}$ & $3.5(2.0-6.2)$ & $5.1(2.7-9.8)^{*}$ \\
\hline & & $>2 \mathrm{~cm}$ & 54 & $1.2(0.7-2.1)$ & $1.5(0.8-2.6)$ & $1.1(0.6-2.0)$ & $1.9(1.0-3.7)$ \\
\hline & \multirow{2}{*}{$>52$} & $\leq 2 \mathrm{~cm}$ & 11 & $0.6(0.1-2.7)$ & $0.6(0.1-2.9)$ & $0.6(0.1-2.7)$ & $0.9(0.1-5.0)$ \\
\hline & & $>2 \mathrm{~cm}$ & 12 & $0.8(0.2-2.4)$ & $0.9(0.3-2.9)$ & $0.9(0.3-2.8)$ & $2.4(0.6-9.3)$ \\
\hline \multicolumn{8}{|l|}{$B R C A 2^{2}$} \\
\hline \multirow{5}{*}{$\begin{array}{l}\text { BRCA } 1 \text { (only grade } \\
3 \text { tumours included }\end{array}$} & \multicolumn{7}{|c|}{ Cases stratified by year of diagnosis and tumour diameter } \\
\hline & \multirow{2}{*}{$\leq 1995$} & $\leq 2 \mathrm{~cm}$ & 53 & $0.8(0.4-1.6)$ & $x^{2}$ & $0.9(0.4-1.7)$ & $x^{2}$ \\
\hline & & $>2 \mathrm{~cm}$ & 37 & $0.5(0.3-1.1)$ & $0.8(0.4-1.8)$ & $1.0(0.5-1.9)$ & $2.4(1.1-5.3)^{*}$ \\
\hline & \multirow{2}{*}{ >1995 } & $\leq 2 \mathrm{~cm}$ & 48 & $5.7(3.0-10.7)$ & $5.8(3.0-11.2)^{*}$ & $6.5(3.4-12.6)$ & $10.3(4.8-22.3)^{*}$ \\
\hline & & $>2 \mathrm{~cm}$ & 63 & $1.0(0.6-1.8)$ & $1.0(0.6-1.8)$ & $0.9(0.5-1.6)$ & $1.5(0.8-2.8)$ \\
\hline$B R C A 2^{2}$ & & & & & & & \\
\hline
\end{tabular}

${ }^{1}$ Model with surgery (modified radical mastectomy versus breast conserving therapy) adjusted for: differentiation grade (grade 1 \& 2 (ref), grade 3 and missing), nodal status (node positive (ref), node negative and missing), age at breast cancer incidence ( $<35$ years, $35-52$ years (ref) and $>52$ years, tumour diameter ( $\leq 2 \mathrm{~cm}$ (ref), $>2 \mathrm{~cm}$, missing). All models were adjusted for the above-mentioned factors, unless the model was stratified for that factor.

${ }^{2}$ Insufficient cases to run the model.

* Statistically significant $(\mathrm{P}<0.05)$ (indicated only for adjusted ORs).

that found that of 66 studies assessing the survival of BRCA1/2 mutation carriers, only 8 corrected for confounding by adjuvant treatment [32]. Current evidence suggests that contrary to currently held beliefs, if confounding by treatment is taken into account, differences in survival between BRCA1/2 mutation carriers and sporadic breast cancer patients if any are likely to be small.

The Dutch guidelines for breast cancer treatment do not differ specifically for BRCA1/2 cases and sporadic breast cancer cases. Breast conserving surgery was introduced in 1986 following the EORTC 10,801 trial [12]. Age by itself and tumor size have been shown to be important factors in the choice of type of breast cancer surgery, but even within the Netherlands there is variance between hospitals [33]. In our analysis, we found an increased probability of more extensive surgery in BRCA1/2 cases with a tumor $\leq 2 \mathrm{~cm}$, both compared to the NKI-AVL cancer hospital-based (where $22 \%$ of the BRCA1/2 cases had also been treated) as well as to the 
Table 4. Odds of receiving chemotherapy for BRCA1/2 cases compared to population-based and cancer hospital-based cases.

\begin{tabular}{|c|c|c|c|c|c|c|c|}
\hline \multirow{3}{*}{ Subgroup } & & & \multirow{3}{*}{$\mathrm{N}$} & \multicolumn{2}{|c|}{$\begin{array}{l}B R C A 1 / 2 \text { cases versus } \\
\text { population-based cases }\end{array}$} & \multicolumn{2}{|c|}{$\begin{array}{l}B R C A 1 / 2 \text { cases versus } \\
\text { hospital-based cases }\end{array}$} \\
\hline & & & & Unadjusted & Adjusted $^{1}$ & Unadjusted & Adjusted $^{1}$ \\
\hline & & & & OR (95\%-CI) & OR (95\%-CI) & OR (95\%-CI) & OR (95\%-CI) \\
\hline \multirow{3}{*}{ BRCA 1} & \multicolumn{7}{|c|}{ Cases stratified by nodal status } \\
\hline & \multicolumn{2}{|c|}{ Negative } & 223 & $8.8(6.4-12.1)$ & $1.9(1.3-2.7)^{*}$ & $8.8(6.2-12.4)$ & $2.5(1.7-3.8)^{*}$ \\
\hline & \multicolumn{2}{|c|}{ Positive } & 115 & $9.6(5.9-15.6)$ & $4.3(2.4-7.6)^{*}$ & $6.3(3.9-10.2)$ & $2.7(1.4-5.3)^{*}$ \\
\hline \multirow{2}{*}{ BRCA 2} & \multicolumn{2}{|c|}{ Negative } & 49 & $2.6(1.0-6.6)$ & $0.9(0.3-2.4)$ & $2.6(1.0-6.6)$ & $1.2(0.5-3.4)$ \\
\hline & \multicolumn{2}{|c|}{ Positive } & 56 & $9.3(4.7-18.4)$ & $4.4(2.0-9.7)^{*}$ & $6.1(3.1-12.1)$ & $2.7(1.1-6.5)^{*}$ \\
\hline \multirow{4}{*}{$\begin{array}{c}\text { ER-negative only } \\
B R C A 1 / 2 \text { combined }\end{array}$} & \multicolumn{2}{|c|}{ Negative } & 142 & $4.6(3.1-6.8)$ & $2.0(1.3-3.1)^{*}$ & $24.3(15.2$ - 38.9) & $x^{b}$ \\
\hline & \multicolumn{2}{|c|}{ Positive } & 65 & $8.4(4.1-17.1)$ & $3.8(1.8-8.3)^{*}$ & $8.4(4.1-17.0)$ & $2.6(1.0-6.7)$ \\
\hline & \multicolumn{7}{|c|}{ Cases stratified by age at breast cancer diagnosis } \\
\hline & \multicolumn{2}{|r|}{$<35$} & 108 & $1.6(1.1-2.5)$ & $3.2(1.8-5.8)^{*}$ & $1.1(0.7-1.7)$ & $2.4(1.2-4.7)^{*}$ \\
\hline \multirow[t]{3}{*}{ BRCA 1} & \multicolumn{2}{|r|}{$35-52$} & 205 & $1.6(1.2-2.2)$ & $2.5(1.8-3.6)^{*}$ & $1.0(0.8-1.3)$ & $2.5(1.6-3.7)^{*}$ \\
\hline & \multicolumn{2}{|r|}{$>52$} & 44 & $5.4(2.7-10.7)$ & $3.7(1.7-8.0)^{*}$ & $6.0(3-12.1)$ & $7.8(3.5-17.7)^{*}$ \\
\hline & \multicolumn{2}{|r|}{$<35$} & 16 & $1.2(0.4-3.2)$ & $1.0(0.3-3.3)$ & $0.8(0.3-2.2)$ & $0.3(0.1-1.1)$ \\
\hline \multirow[t]{2}{*}{ BRCA 2} & \multicolumn{2}{|r|}{$35-52$} & 76 & $2.3(1.5-3.6)$ & $2.5(1.4-4.5)^{*}$ & $1.4(0.9-2.2)$ & $1.9(1.0-3.7)$ \\
\hline & \multicolumn{2}{|r|}{$>52$} & 19 & $5.4(2.7-10.7)$ & $3.5(1.0-12.2)$ & $4.8(1.6$ - 14.6) & $7.4(2.1-26.0)^{*}$ \\
\hline \multirow{3}{*}{$\begin{array}{c}\text { ER-negative only } \\
\text { BRCA } 1 / 2 \text { combined }\end{array}$} & \multicolumn{2}{|r|}{$<35$} & 69 & $2.0(1.1-3.6)$ & $3.8(1.8-8.2)^{*}$ & $1.3(0.7-2.5)$ & $3.8(1.2-12.1)^{*}$ \\
\hline & \multicolumn{2}{|r|}{$35-52$} & 122 & $1.5(1.0-2.2)$ & $2.1(1.3-3.3)^{*}$ & $1.3(0.9-1.8)$ & $4.1(2.3-7.3)^{*}$ \\
\hline & \multicolumn{2}{|r|}{$>52$} & 20 & $2.4(0.9-6.2)$ & $2.7(1.0-7.7)$ & $8.6(3.2-22.8)$ & $39.1(8.9-173)^{*}$ \\
\hline \multirow{5}{*}{$\begin{array}{l}\text { BRCA 1(only grade } \\
3 \text { tumours included) }\end{array}$} & \multicolumn{7}{|c|}{ Cases stratified by year of diagnosis and nodal status } \\
\hline & \multirow{2}{*}{$\leq 1995$} & Negative & 64 & $2.6(0.8-9.0)$ & $x^{2}$ & $1.9(0.6-5.9)$ & $x^{2}$ \\
\hline & & Positive & 28 & $11.2(4.1-30.3)$ & $10.3(2.3-46.7)^{*}$ & $7.1(2.7-18.9)$ & $8.6(1.8-40.6)^{*}$ \\
\hline & $>1995$ & Negative & 68 & $7.8(4.5-13.5)$ & $x^{2}$ & $4.3(2.4-7.8)$ & $2.8(1.5-5.2)^{*}$ \\
\hline & $>1995$ & Positive & 37 & $12.9(3.9-42.3)$ & $6.6(1.9-22.3)^{*}$ & $6.7(2-22.2)$ & $x^{2}$ \\
\hline$B R C A 2^{b}$ & & & & & & & \\
\hline & $<1905$ & Negative & 62 & $4.5(1.1-18.3)$ & $1.2(0.2-5.9)$ & $4.8(1.3-18.2)$ & $x^{2}$ \\
\hline ER-negative only BRCA & $=1993$ & Positive & 27 & $8.5(3.2-22.9)$ & $3.8(1.1-13.0)^{*}$ & $7.0(2.6-18.5)$ & $1.9(0.5-7.1)$ \\
\hline $1 / 2$ combined & 5 & Negative & 80 & $5.8(3.5-9.6)$ & $3.0(1.7-5.2)^{*}$ & $38.8(21.5$ - 70.2) & $x^{2}$ \\
\hline & -1995 & Positive & 38 & $8.3(2.9-23.7)$ & $5.0(1.7-14.9)^{*}$ & $9.9(3.5$ - 28.1) & $x^{2}$ \\
\hline
\end{tabular}

${ }^{1}$ Model with surgery (modified radical mastectomy versus breast conserving therapy) adjusted for: differentiation grade (grade $1 \& 2$ (ref), grade 3 and missing), nodal status (node positive (ref), node negative and missing), age at breast cancer incidence $(<35$ years, $35-52$ years (ref) and $>52$ years, tumour diameter ( $\leq 2 \mathrm{~cm}$ (ref), $>2 \mathrm{~cm}$, missing). All models were adjusted for the above-mentioned factors, unless the model was stratified for that factor.

${ }^{2}$ Insufficient cases to run the model.

${ }^{*}$ Statistically significant $(\mathrm{P}<0.05)$ (indicated only for adjusted ORs).

population-based reference group. It seemed that particularly after 1995, the BRCA1/2 cases were more likely to receive a modified radical mastectomy for smaller tumors, but we lacked power to investigate the effects of knowledge of BRCA-carriership. It is known that a part of the affected BRCA1/2 cases also choose for a contralateral preventive mastectomy (in combination with a modified radical mastectomy of the affected breast) to prevent contralateral breast cancer [20], which might play a role in the higher rate of modified radical mastectomies observed in BRCA1/2 cases in this study. Unfortunately, in the current study it is unknown how many BRCA1/2 cases underwent a contralateral mastectomy. Our observations also may reflect the (early) perception of many physicians that more extensive surgery for mutation carriers would be better in the long term. Possibly, effects of a general time trend of increased use of modified radical mastectomy in patients $<50$ years also played a role [33]. 
Both node positive and node negative BRCA1/2 cases were more likely to receive chemotherapy for ER-negative tumors. Until the publication of the EBCTCG review results in 1997 [14] and the implementation of the first Dutch guidelines on adjuvant systemic breast cancer treatment in 1998, adjuvant systemic treatment was rarely used in node-negative breast cancer in the Netherlands. Since then adjuvant systemic therapy in node negative patients has become more commonplace and included in the guideline for those patients from whom a 10 -year survival gain of at least $3 \%-5 \%$ is expected [13] [14]. This might partly explain our observation that node negative BRCA1/2 cases were more likely to receive chemotherapy especially after 1998. Another explanation for this observation might be the growing awareness that BRCA-associated breast cancer frequently metastasized and was possibly more sensitive to chemotherapy [34] [35].

The current study confirmed the high prevalence of high-grade tumors in BRCA1 and BRCA2 cases (88\% and $69 \%$ ) described in the literature [9]. Nowadays differentiation grade is a factor of consideration regarding the use of adjuvant systemic therapy in node-negative breast cancer given the fact that the cumulative 10-year survival of high grade tumors has been estimated to be $30 \%$ - 78\% compared to $90 \%$ - $94 \%$ for breast cancers with the lowest differentiation grade tumors [4]. However, in the current Dutch treatment guidelines a high-grade tumor alone is not considered sufficient justification for prescribing adjuvant systemic therapy [13], and effects we found were consistent also when only comparing grade 3 tumors. Moreover, in the period that the majority of the included cases were diagnosed, grade was not yet a relevant factor used in clinical decision-making. Unfortunately, we do not have information on other tumor markers such as EGFR, E-cadherin and ki67. Also, the majority of patients were treated before the nationwide introduction of Trastuzumab.

Despite the unique results of the current study, we are aware of some limitations. Ideally we would have compared BRCA1/2 cases to sporadic cases from the same hospitals. Young women ( $<40$ years) treated at a general hospital were more likely to undergo breast conserving therapy compared to those treated at a teaching or academic hospital [36]. Also, a pronounced difference in the use of adjuvant systemic therapy at a hospital level was found though this did not appear to be associated with type of hospital [36]. The variations in treatment observed could be due to a delayed introduction of new techniques or implementation of new scientific insights. Importantly, overall we observed similarly increased odds, or at least overlapping confidence intervals, for the BRCA1/2 cases for receiving more extensive treatment compared to both the population-based and the cancer hospital-based reference groups. Secondly, the current multicenter study included a sizeable study population and had a long follow up; the treatment data is largely complete, yet a large proportion of tumor characteristics, such as tumor differentiation grade and ER-status are missing, partly due to the work-up and the factors considered during the period of treatment. Also, possibly just a few of the 59 obligate BRCA1/2 carriers (obligate based on mendelian inheritance) might have turned out to be BRCA1/2 mutation negative would they have been tested individually. Further, since no testing was performed in the reference population, there might have been some mutation carriers in these populations. However, it is unlikely that this affected the results given the large number of patients in the reference groups and the small proportion of mutation carriers expected. In addition, selection bias may have occurred as patients who were included in the GEO-HEBON cohort if they had responded to a mailed questionnaire. This could have led to the selection of those in better physical condition or the more motivated patients. If a more extensive treatment has led to a better survival, this is overrepresented in the selected cohort. Further, another draw-back of our study was the small numbers in specific subgroup analyses, producing wide confidence intervals. Also, a proportion of the BRCA1/2 mutation carriers were not aware of their BRCA status at the time of diagnosis and treatment. However, it is worth noting that although they were not aware of their BRCA status, family history was known and was considered an important prognostic factor. Finally, reasons driving treatment decisions were not clearly identifiable in this study, given the retrospective study design.

Currently, as far as we know, there is little information available regarding the influence of having breast cancer in the family or being a BRCA1/2 mutation carrier on the clinical breast cancer treatment decision process. Most studies evaluated factors influencing the decision whether or not to undergo additional preventive surgery in high risk populations, showing that BRCA test results are one of the most important factors in combination with the carrier's age and personal circumstances (e.g. marital status, having children) for this decision [19]. It is unknown whether these factors also play a role in decisions on breast cancer treatment for BRCA1/2 mutation carriers, additionally, we do not know whether the differences in treatment for BRCA1/2 cases observed in this study were driven by the choice of the patient or the physician. Over the last decades shared decision-making has been promoted in clinical practice and a large proportion of patients favors this trend [37]. Yet, exploration 
of whether breast cancer surgeons and oncologists who shared decision-making with their patients, felt comfortable with this approach, and whether they perceived any barriers to implementation, showed a substantial gap between the high self-reported comfort levels with shared decision-making (87\% - 89\%) and the self- reported use (56\% - 69\%) [38] [39]. The physicians reported that time constraints and patient's knowledge and psychological state were the most important factors inhibiting shared decision-making [38] [39].

\section{Conclusion}

In conclusion, we found evidence of different breast cancer treatment strategies in BRCA1/2-associated compared to sporadic cases with similar tumor characteristics, including more mastectomies and administration of adjuvant chemotherapy among $B R C A 1 / 2$ cases. Although it is unknown which factors exactly played a role in the treatment decision among BRCA1/2 mutation carriers and sporadic patients, respectively, and whether treatment choice was driven by the patient or the physician, the results of the current analyses highlight the importance of the need to be aware of such differences in daily practice and interpretation of survival studies on BRCA1/2 mutation carriers.

\section{Acknowledgements}

We would like to thank all patients and Esther Janssen, Kiki Jeanson, GittyJaanen, Petra Bos, Jannet Blom and Twiggy van Cronenburg for their assistance with data collection.

\section{Authors' Contributions}

MKS, MJH and CS designed the study; EGE, MKS, MJH, MK, CJvA, MGEMA, LVvdPF, SM and MAR collected the data; EGE and MKS performed the data analyses; EGE, MKS, MJH, MK and CS interpreted the data and wrote the paper; EGE, MKS, MK, MJH, CS, RAEMT, CJvA, MGEMA, LVvdPF, SM, SV, MAR read and approved the final version of the manuscript.

\section{Funding}

Dutch Cancer Society grants NKI2009-4363 and DDHK 2009-4318; NWO 184.021.007 (BBMRI-NL); Netherlands Organization of Scientific Research grant NWO/Zon-MW 91109024 (HEBON Resource).

\section{Conflict of Interest}

The authors declare that they have no conflict of interest.

\section{Full List of HEBON Collaborators and Affiliations}

The Hereditary Breast and Ovarian Cancer Research Group Netherlands (HEBON) consists of the following Collaborating Centers: Coordinating center: Netherlands Cancer Institute, Amsterdam, NL: M.A. Rookus, F.B.L. Hogervorst, F.E. van Leeuwen, S. Verhoef, M.K. Schmidt, J.L. de Lange; Erasmus Medical Center, Rotterdam, NL: J.M. Collée, A.M.W. van den Ouweland, M.J. Hooning, C. Seynaeve, C.H.M. van Deurzen, I.M. Obdeijn; Leiden University Medical Center, NL: C.J. van Asperen, J.T. Wijnen, R.A.E.M. Tollenaar, P. Devilee, T.C.T.E.F. van Cronenburg; Radboud University Nijmegen Medical Center, NL: C.M. Kets, A.R. Mensenkamp; University Medical Center Utrecht, NL: M.G.E.M. Ausems, R.B. van der Luijt; Amsterdam Medical Center, NL: C.M. Aalfs, T.A.M. van Os; VU University Medical Center, Amsterdam, NL: J.J.P. Gille, Q. Waisfisz, H.E.J. Meijers-Heijboer; University Hospital Maastricht, NL: E.B. Gómez-Garcia, M.J. Blok; University Medical Center Groningen, NL: J.C. Oosterwijk, A.H. van der Hout, M.J. Mourits, G.H. de Bock. The Netherlands Foundation for the detection of hereditary tumours, Leiden, NL: H.F. Vasen.

\section{References}

[1] Antoniou, A., Pharoah, P., Narod, S., Risch, H., Eyfjord, J., Hopper, J., et al. (2003) Average Risks of Breast and Ovarian Cancer Associated with BRCA1 or BRCA2 Mutations Detected in Case Series Unselected for Family History: A Combined Analysis of 22 Studies. The American Journal of Human Genetics, 72, 1117-1130.

http://dx.doi.org/10.1086/375033 
[2] Kiemeney, L., Lemmers, F., Verhoeven, R., Aben, K., Honing, C., de Nooijer, J., et al. (2008) De kans op kanker voor Nederlanders. Nederlands Tijdschrift voor Geneeskunde, 152, 2233-2241.

[3] Boyle, P. and Levin, B., Eds. (2008) World Cancer Report 2008. International Agency for Research on Cancer (IARC), Geneva, 1-6-2010.

[4] Soerjomataram, I., Louwman, M., Ribot, J., Roukema, J. and Coebergh, J. (2008) An Overview of Prognostic Factors for Long-Term Survivors of Breast Cancer. Breast Cancer Research and Treatment, 107, 309-330. http://dx.doi.org/10.1007/s10549-007-9556-1

[5] Brekelmans, C., Seynaeve, C., Menke-Pluymers, M., Brüggenwirth, H., Tilanus-Linthorst, M., Bartels, C., et al. (2006) Survival and Prognostic Factors in BRCA1-Associated Breast Cancer. Annals of Oncology, 17, 391-400. http://dx.doi.org/10.1093/annonc/mdj095

[6] Foulkes, W., Reis-Filho, J. and Narod, S. (2010) Tumor Size and Survival in Breast Cancer-A Reappraisal. Nature Reviews Clinical Oncology, 7, 348-353. http://dx.doi.org/10.1038/nrclinonc.2010.39

[7] Tutt, A., Robson, M., Garber, J., Domchek, S., Audeh, M., Weitzel, J., et al. (2010) Oral Poly(ADP-Ribose) Polymerase Inhibitor Olaparib in Patients with BRCA1 or BRCA2 Mutations and Advanced Breast Cancer: A Proof-of-Concept Trial. The Lancet, 376, 235-244. http://dx.doi.org/10.1016/S0140-6736(10)60892-6

[8] Bordeleau, L., Panchal, S. and Goodwin, P. (2010) Prognosis of BRCA-Associated Breast Cancer: A Summary of Evidence. Breast Cancer Res Treat, 119, 13-24. http://dx.doi.org/10.1007/s10549-009-0566-Z

[9] Lee, E., Park, S., Park, B., Kim, S., Lee, M., Ahn, S., et al. (2010) Effect of BRCA1/2 Mutation on Short-Term and Long-Term Breast Cancer Survival: A Systematic Review and Meta-Analysis. Breast Cancer Research and Treatment, 122, 11-25. http://dx.doi.org/10.1007/s10549-010-0859-2

[10] Goldhirsch, A., Ingle, J., Gelber, R., Coates, A., Thürlimann, B., Senn, H., et al. (2009) Thresholds for Therapies: Highlights of the St Gallen International Expert Consensus on the Primary Therapy of Early Breast Cancer 2009. Annals of Oncology, 20, 1319-1329. http://dx.doi.org/10.1093/annonc/mdp322

[11] Maughan, K., Lutterbie, M. and Ham, P. (2010) Treatment of Breast Cancer. American Family Physician, 81, 13391346.

[12] Litiere, S., Werutsky, G., Fentiman, I.S., Rutgers, E., Christiaens, M.R., Van Limbergen, E., et al. (2012) Breast Conserving Therapy versus Mastectomy for Stage I-II Breast Cancer: 20 Year Follow-Up of the EORTC 10801 Phase 3 Randomised Trial. The Lancet Oncology, 13, 412-419. http://dx.doi.org/10.1016/S1470-2045(12)70042-6

[13] NABON (2012) Breast Cancer, Dutch Guideline, Version 2.0. http://www.oncoline.nl/mammacarcinoom

[14] Early Breast Cancer Trialists' Collaborative Group (1998) Polychemotherapy for Early Breast Cancer: An Overview of the Randomised Trials. The Lancet, 352, 930-942. http://dx.doi.org/10.1016/S0140-6736(98)03301-7

[15] Vervoort, M., Draisma, G., Fracheboud, J., Poll-Franse, L. and de Koning, H. (2004) Trends in the Usage of Adjuvant Systemic Therapy for Breast Cancer in the Netherlands and Its Effect on Mortality. British Journal of Cancer, 91, 242247. http://dx.doi.org/10.1038/sj.bjc.6601969

[16] Vos, E., Linn, S. and Rodenhuis, S. (2006) Effects and Costs of Adjuvant Chemotherapy for Operable Lymph Node Positive Breast Cancer with HER2/Neu Overexpression. Nederlands Tijdschrift voor Geneeskunde, 150, 776-780.

[17] Early Breast Cancer Trialists' Collaborative Group (2005) Effects of Chemotherapy and Hormonal Therapy for Early Breast Cancer on Recurrence and 15-Year Survival: An Overview of the Randomised Trials. The Lancet, 365, 16871717. http://dx.doi.org/10.1016/S0140-6736(05)66544-0

[18] Devilee, P., Tollenaar, R. and Cornelisse, C. (2012) From Gene to Disease; from BRCA1 or BRCA2 to Breast Cancer. Nederlands Tijdschrift voor Geneeskunde, 144, 2549-2551.

[19] Klitzman, R. and Chung, W. (2010) The Process of Deciding about Prophylactic Surgery for Breast and Ovarian Cancer: Patient Questions, Uncertainties, and Communication. American Journal of Medical Genetics, 152A, 52-66. http://dx.doi.org/10.1002/ajmg.a.33068

[20] Meijers-Heijboer, H., Brekelmans, C., Menke-Pluymers, M., Seynaeve, C., Baalbergen, A., Burger, C., et al. (2003) Use of Genetic Testing and Prophylactic Mastectomy and Oophorectomy in Women with Breast or Ovarian Cancer from Families with a BRCA1 or BRCA2 Mutation. Journal of Clinical Oncology, 21, 1675-1681.

[21] Rebbeck, T., Kauff, N. and Domchek, S. (2009) Meta-Analysis of Risk Reduction Estimates Associated with RiskReducing Salpingo-Oophorectomy in BRCA1 or BRCA2 Mutation Carriers. Journal of the National Cancer Institute, 101, 80-87. http://dx.doi.org/10.1093/jnci/djn442

[22] Domchek, S., Friebel, T., Neuhausen, S., Wagner, T., Evans, G., Isaacs, C., et al. (2006) Mortality after Bilateral Salpingo-Oophorectomy in BRCA1 and BRCA2 Mutation Carriers: A Prospective Cohort Study. The Lancet Oncology, 7, 223-229. http://dx.doi.org/10.1016/S1470-2045(06)70585-X

[23] Rebbeck, T., Friebel, T., Lynch, H., Neuhausen, S., van’t Veer, L., Garber, J., et al. (2004) Bilateral Prophylactic Mas- 
tectomy Reduces Breast Cancer Risk in BRCA1 and BRCA2 Mutation Carriers: The PROSE Study Group. Journal of Clinical Oncology, 22, 1055-1062.

[24] Graves, K., Peshkin, B., Halbert, C., DeMarco, T., Isaacs, C. and Schwartz, M. (2007) Predictors and Outcomes of Contralateral Prophylactic Mastectomy among Breast Cancer Survivors. Breast Cancer Research and Treatment, 104, 321-329. http://dx.doi.org/10.1007/s10549-006-9423-5

[25] Domchek, S., Friebel, T. and Singer, C. (2010) Association of Risk-Reducing Surgery in BRCA1 or BRCA2 Mutation Carriers with Cancer Risk and Mortality. Japan Automobile Manufacturers Association, 304, 967-975. http://dx.doi.org/10.1001/jama.2010.1237

[26] Klaren, H., van't Veer, L., van Leeuwen, F. and Rookus, M. (2003) Potential for Bias in Studies on Efficacy of Prophylactic Surgery for BRCA1 and BRCA2 Mutation. Journal of the National Cancer Institute, 95, 941-947. http://dx.doi.org/10.1093/jnci/95.13.941

[27] Pijpe, A., Manders, P., Brohet, R., Collée, J., Verhoef, S., Vasen, H., et al. (2010) Physical Activity and the Risk of Breast Cancer in BRCA1/2 Mutation Carriers. Retreats-Breast Cancer Recovery, 120, 235-244. http://dx.doi.org/10.1007/s10549-009-0476-0

[28] Sukel, M., van de Poll-Franse, L., Nieuwenhuijzen, G., Vreugdenhil, G., Herings, R., Coebergh, J., et al. (2008) Substantial Increase in the Use of Adjuvant Systemic Treatment for Early Stage Breast Cancer Reflects Changes in Guidelines in the Period 1990-2006 in the Southeastern Netherlands. European Journal of Cancer, 44, 1846-1854. http://dx.doi.org/10.1016/j.ejca.2008.06.001

[29] Mook, S., Schmidt, M., Rutgers, E., van de Velde, A., Visser, O., Rutgers, S., et al. (2009) Calibration and Discriminatory Accuracy of Prognosis Calculation for Breast Cancer with the Online Adjuvant! Program: A Hospital-Based Retrospective Cohort Study. The Lancet Oncology, 10, 1070-1076. http://dx.doi.org/10.1016/S1470-2045(09)70254-2

[30] Bontenbal, M., van Putten, W., Burghouts, J., Baggen, M., Ras, G., Stiegelis, W., et al. (2000) Value of Estrogenic Recruitment before Chemotherapy: First Randomized Trial in Primary Breast Cancer. Journal of Clinical Oncology, 18, 734.

[31] Council of the Dutch Federation of Medical Scientific Societies Code of Conduct for Medical Research. http://www.federa.org/sites/default/files/bijlagen/coreon/code of conduct for medical research 1.pdf

[32] van den Broek, A., Schmidt, M., van’t Veer, L., Tollenaar, R. and van Leeuwen, F (2015) Worse Breast Cancer Prognosis BRCA1/BRCA2 Mutation Carriers: What's the Evidence? A Systematic Review with Meta-Analysis. PLoS ONE, 10, e0120189. http://dx.doi.org/10.1371/journal.pone.0120189

[33] Siesling, S., van de Poll-Franse, L.V., Jobsen, J.J., Repelaer van Driel, O.J. and Voogd, A.C. (2005) Trends and Variation in Breast Conserving Surgery in the Southeast and East of the Netherlands over the Period 1990-2002. Nederlands Tijdschrift voor Geneeskunde, 149, 1941-1946.

[34] Bayraktar, S. and Glück, S. (2012) Systemic Therapy Options in BRCA Mutation-Associated Breast Cancer. Breast Cancer Research and Treatment, 135, 355-366. http://dx.doi.org/10.1007/s10549-012-2158-6

[35] Kriege, M., Seynaeve, C., Meijers-Heijboer, H., Collee, J., Menke-Pluymers, M., Bartels, C., et al. (2009) Sensitivity to First-Line Chemotherapy for Metastatic Breast Cancer in BRCA1 and BRCA2 Mutation Carriers. Journal of Clinical Oncology, 27, 3764-3771.

[36] van Steenbergen, L., Poll-Franse, L., Wouters, M., Jansen-Landheer, M., Coebergh, J., Struikmans, H., et al. (2010) Variation in Management of Early Breast Cancer in the Netherlands, 2003-2006. European Journal of Surgical Oncology, 36, S36-S43. http://dx.doi.org/10.1016/j.ejso.2010.06.021

[37] Degner, L., Kristjanson, L., Bowman, D., Sloan, J., Carriere, K., O’Neil, J., et al. (1997) Information Needs and Decisional Preferences in Women with Breast Cancer. Journal of the American Medical Association, 277, 1485-1492. http://dx.doi.org/10.1001/jama.1997.03540420081039

[38] Step, M., Siminoff, L. and Rose, J. (2009) Differences in Oncologist Communication across Age Groups and Contributions to Adjuvant Decision Outcomes. Journal of the American Geriatrics Society, 57, S279-S282. http://dx.doi.org/10.1111/j.1532-5415.2009.02512.x

[39] Charles, C., Gafni, A. and Whelan, T. (2004) Self-Reported Use of Shared Decision-Making among Breast Cancer Specialists and Perceived Barriers and Facilitators to Implementing This Approach. Health Expectations, 7, 338-348. http://dx.doi.org/10.1111/j.1369-7625.2004.00299.x 